\title{
A CLASS OF REACTION-DIFFUSION SYSTEMS WITH NONLOCAL INITIAL CONDITIONS*
}

\author{
BY \\ MONICA-DANA BURLICĂ and DANIELA ROŞU
}

\begin{abstract}
We consider an abstract nonlinear multi-valued reaction-diffusion system with delay and, using some compactness arguments coupled with metric fixed point techniques, we prove some sufficient conditions for the existence of at least one $\mathcal{C}^{0}$-solution.

Mathematics Subject Classification 2010: 34G25, 34K30, 35K55, 35K57, 47H06.

Key words: differential delay evolution systems, nonlocal delay initial condition, metric fixed point arguments, non-resonance condition, nonlinear reaction-diffusion systems.
\end{abstract}

\section{Introduction}

The goal of this paper is to provide an existence result for $C^{0}$-solutions to the following class of nonlinear delay differential reaction-diffusion systems with nonlocal initial conditions:

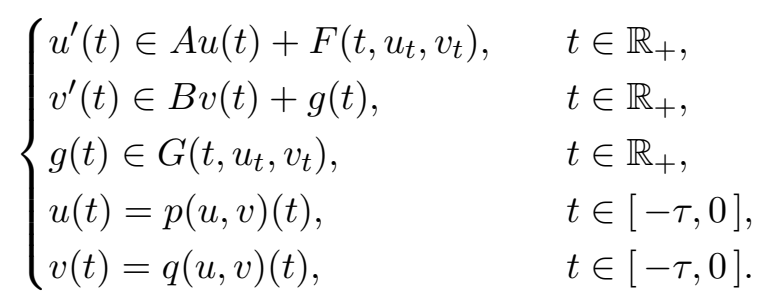

Here $X$ and $Y$ are Banach spaces, $A: D(A) \subseteq X \rightsquigarrow X$ and $B: D(B) \subseteq$ $Y \rightsquigarrow Y$ are the infinitesimal generators of two nonlinear semigroups of contractions, $\tau>0$ is arbitrary fixed, $F: \mathbb{R}_{+} \times C([-\tau, 0] ; \overline{D(A)}) \times C([-\tau, 0]$;

\footnotetext{
${ }^{*}$ This work was supported by a grant of the Romanian National Authority for Scientific Research, CNCS-UEFISCDI, project number PN-II-ID-PCE-2011-3-0052.
} 
$\overline{D(B)}) \rightarrow X$ is a continuous function while $G: \mathbb{R}_{+} \times C([-\tau, 0] ; \overline{D(A)}) \times$ $C([-\tau, 0] ; \overline{D(B)}) \rightsquigarrow Y$ is a strongly-weakly u.s.c. multi-function with nonempty, convex and weakly compact values. The nonlocal functions $p: C_{b}([-\tau,+\infty) ; \overline{D(A)}) \times C_{b}([-\tau,+\infty) ; \overline{D(B)}) \rightarrow C([-\tau, 0] ; \overline{D(A)})$ and $q: C_{b}([-\tau,+\infty) ; X) \times C_{b}([-\tau,+\infty) ; \overline{D(B)}) \rightarrow C([-\tau, 0] ; \overline{D(B)})$ are nonexpansive.

If $X$ is a Banach space and $a$ is a real fixed number, we denote by $C_{b}([a,+\infty) ; X)$ the space of $X$-valued bounded and continuous functions on $[a,+\infty)$, with the sup-norm $\|\cdot\|_{C_{b}([a,+\infty) ; X)}=\sup \{\|u(t)\|, t \in[a,+\infty)\}$.

The closed subset in $C_{b}([a,+\infty) ; X)$ consisting of all elements $u \in$ $C_{b}([a,+\infty) ; X)$ satisfying $u(t) \in \overline{D(A)}$, for each $t \in[a,+\infty)$, is denoted by $C_{b}([a,+\infty) ; \overline{D(A)})$. If $[a, b]$ is a real compact interval we denote by $C([a, b] ; X)$ the space of all continuous functions from $[a, b]$ to $X$ endowed with the sup-norm $\|\cdot\|_{C([a, b] ; X)}$ and by $C([a, b] ; \overline{D(A)})$ the subset which contains the functions $u \in C([a, b] ; X)$ with $u(t) \in \overline{D(A)}$ for each $t \in[a, b]$.

If $u \in C([-\tau,+\infty) ; X)$ and $t \in[0,+\infty)$, the delayed function $u_{t} \in$ $C([-\tau, 0] ; X)$ is defined by $u_{t}(s):=u(t+s)$ for each $s \in[-\tau, 0]$. Similarly, we define $C_{b}([a,+\infty) ; Y), C_{b}([a,+\infty) ; \overline{D(B)}), C([a, b] ; \overline{D(B)})$ and the function $v_{t}$.

In the present paper we prove an existence result for a class of nonlinear multi-valued reaction-diffusion system with delay and nonlocal initial conditions. For other results concerning reaction-diffusion systems we mention: Burlic $\breve{~[5], ~ B u r l i c a ̆ ~ a n d ~ R o s ̧ u ~[6], ~[7], ~ D i ́ a z ~ a n d ~ V r a b i e ~[12], ~ N e c u l a ~}$ and VRabie [17], Roşu [19], [20] and the references therein. For (nondelayed) evolution equations subjected to nonlocal initial conditions see PAICU and VRABIE [18] and the references therein. For delay evolution equations with nonlocal initial data see Burlic $\breve{~ a n d ~ R o s ̧ u ~[8] ~ a n d ~ V R A B I E ~[22], ~[23] ~}$ and [25]. The single valued system was considered by Burlic $\breve{A}$, Roşu and

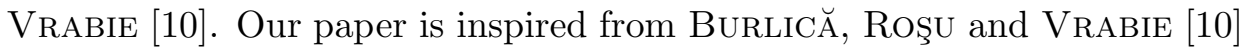
and based on the result of BURLICĂ and Roşu [8] and VRABIE [23] and our main Theorem is adapted to the abstract multi-valued reaction-diffusion systems.

The paper is divided into 5 sections. In Section 2 we recall some useful results throughout the paper. In Section 3 we formulate the main result, i.e. Theorem 3.1. In Section 4 we give some auxiliary lemmas and we prove the main result. In the last Section 5 we give an example referring to the some nonlinear reaction-diffusion system with delay, subjected to nonlocal 
initial conditions: one of the unknown function is subjected to a periodic condition and the other one to a mean condition.

\section{Preliminaries}

We recall some basic concepts and results concerning $m$-dissipative operators and nonlinear evolution equations in Banach spaces. For other definitions, results and details regarding these topics, we refer the reader to BARBU [3] and VRABIE [21]. For more details concerning functional differential equations with delay we refer to Hale [16].

Let $X$ be a Banach space with norm $\|\cdot\|$. An operator $A: D(A) \subseteq$ $X \rightsquigarrow X$ is called dissipative if for each $x_{i} \in D(A)$ and $y_{i} \in A x_{i}, i=1,2$, we have $\left[x_{1}-x_{2}, y_{2}-y_{1}\right]_{+} \geq 0$. Here, $[x, y]_{h}:=\frac{1}{h}(\|x+h y\|-\|x\|)$ and

$$
[x, y]_{+}:=\lim _{h \downarrow 0}[x, y]_{h}=\inf \left\{[x, y]_{h} ; h>0\right\},
$$

for each $x, y \in X$. We remark that $\left|[x, y]_{+}\right| \leq\|y\|$ for each $x, y \in X$. For other properties of the mapping $(x, y) \mapsto[x, y]_{+}$, see BARBU [3, Proposition 3.7, p. 100].

The operator $A: D(A) \subseteq X \rightsquigarrow X$ is called $m$-dissipative if it is dissipative and the range of $I-\lambda A$ is $R(I-\lambda A)=X$, for each $\lambda>0$.

We consider the evolution equation

$$
u^{\prime}(t) \in A u(t)+f(t)
$$

where $f \in L^{1}(a, b ; X)$. A continuous function $u \in C([a, b] ; \overline{D(A)})$ is called $a C^{0}$-solution, or integral solution of $(2.1)$ on $[a, b]$, if it satisfies:

$$
\|u(t)-x\| \leq\|u(s)-x\|+\int_{s}^{t}[u(\tau)-x, f(\tau)+y]_{+} d \tau
$$

for each $x \in D(A), y \in A x$ and $a \leq s \leq t \leq b$.

Theorem 2.1. Let $\omega>0$ and let $A: D(A) \subseteq X \rightsquigarrow X$ be an $m$ dissipative operator such that $A+\omega I$ is dissipative. Then, for each $\xi \in \overline{D(A)}$ and $f \in L^{1}(a, b ; X)$, there exists a unique $C^{0}$-solution of $(2.1)$ on $[a, b]$ which satisfies $u(a)=\xi$. If $f, g \in L^{1}(a, b ; X)$ and $u, v$ are two $C^{0}$-solutions of (2.1) corresponding to $f$ and $g$ respectively, then:

$$
\|u(t)-v(t)\| \leq e^{-\omega(t-s)}\|u(s)-v(s)\|+\int_{s}^{t} e^{-\omega(t-\theta)}\|f(\theta)-g(\theta)\| d \theta
$$


for each $a \leq s \leq t \leq b$.

In particular, if $x \in D(A)$ and $y \in A x$, we have

$$
\|u(t)-x\| \leq e^{-\omega(t-s)}\|u(s)-x\|+\int_{s}^{t} e^{-\omega(t-\theta)}\|f(\theta)+y\| d \theta
$$

for each $a \leq s \leq t \leq b$.

See Barbu [3, Theorem 4.1, p. 128].

For $\xi \in \overline{D(A)}, f \in L^{1}(a, b ; X)$ and $\tau \in[a, b)$, we denote by $u(\cdot, \tau, \xi, f)$ the unique $C^{0}$-solution $u:[\tau, b] \rightarrow \overline{D(A)}$, of the problem (2.1) which satisfies the initial condition $u(\tau)=\xi$. The semigroup generated by $A$ on $\overline{D(A)}$ is denoted by $\{S(t): \overline{D(A)} \rightarrow \overline{D(A)}, t \geq 0\}$ and is defined by $S(t) \xi=u(t, 0, \xi, 0)$ for each $\xi \in \overline{D(A)}$ and $t \geq 0$. We say that the operator $A$ generates a compact semigroup if, for each $t>0$, the operator $S(t)$ is a compact one.

Definition 2.1. The $m$-dissipative operator $A: D(A) \subseteq X \rightsquigarrow X$ is called of complete continuous type if for each $\left(f_{n}\right)_{n}$ in $L^{1}(a, b ; X)$ with $\lim _{n} f_{n}=f$ weakly in $L^{1}(a, b ; X)$ and $\left(u_{n}\right)_{n}$ in $C([a, b] ; \overline{D(A)})$, with $u_{n}$ a $C^{0}$-solution on $[a, b]$ of $u_{n}^{\prime}(t) \in A u_{n}(t)+f_{n}(t), t \in[a, b]$ and $\lim _{n} u_{n}=u$ strongly in $C([a, b] ; X)$, it follows that $u$ is a $C^{0}$-solution on $[a, b]$ of the limit problem $u^{\prime}(t) \in A u(t)+f(t), t \in[a, b]$.

If $A$ generates a compact semigroup and the topological dual of $X$ is uniformly convex or $A$ is linear, then $A$ is of complete continuous type. See VRABIE [21, Corollary 2.3.1, p. 49]. The nonlinear diffusion operator $\Delta \theta$ in $L^{1}(\Omega)$ is an example of a $m$-dissipative operator which generates a compact semigroup and is of complete continuous type in a Banach space whose dual is not uniformly convex. See Theorem 5.1.

Definition 2.2. A subset $\mathcal{F} \subseteq L^{1}(a, b ; X)$ is called uniformly integrable if, for each $\gamma>0$ there exists $\delta(\gamma)>0$ such that

$$
\int_{E}\|f(s)\| d s \leq \gamma
$$

for each $f \in \mathcal{F}$ and each measurable subset $E \subseteq[a, b]$ whose Lebesgue measure satisfies $\lambda(E)<\delta(\gamma)$. 
Remark 2.1. One may easily check out that each uniformly integrable subset in $L^{1}(a, b ; X)$ is norm bounded in $L^{1}(a, b ; X)$. We also remark that if $\mathcal{F} \subseteq L^{1}(a, b ; X)$ is bounded in $L^{p}(a, b ; X)$ for some $p \in(1,+\infty]$, then $\mathcal{F}$ is uniformly integrable.

Theorem 2.2. Let $A: D(A) \subseteq X \rightsquigarrow X$ be m-dissipative operator which generates a compact semigroup. Let $B \subseteq \overline{D(A)}$ be bounded and let $\mathcal{F}$ be uniformly integrable in $L^{1}(a, b ; X)$. Then, for each $c \in(a, b)$, the $C^{0}$-solutions set $\{u(\cdot, a, \xi, f) ; \xi \in B, f \in \mathcal{F}\}$ is relatively compact in $C([c, b] ; X)$. If, in addition, $B$ is relatively compact in $X$, then the $C^{0}$-solutions set is relatively compact even in $C([a, b] ; X)$.

See Baras [2] and VRABIE [21, Theorems 2.3.2 and 2.3.3, p. 46-47].

Now, we recall a version of a general fixed point theorem, for multifunctions, due to GLICKSBERG [15] in a locally convex space.

Theorem 2.3. Let $K$ be a nonempty, convex and compact set in a separated locally convex space and let $Q: K \rightsquigarrow K$ be a nonempty, closed and convex valued multi-function with closed graph. Then $Q$ has at least one fixed point, i.e. there exists $f \in K$ such that $f \in Q(f)$.

\section{The main result}

Let $a \in \mathbb{R}$. On the space $C_{b}([a,+\infty) ; Y)$ we consider the family of seminorms $\left\{\|\cdot\|_{k} ; k \in \mathbb{N}, k>a\right\}$, defined by $\|v\|_{k}=\|v\|_{C([a, k] ; Y)}$ which generates a locally convex and separated topology. This space will be denoted by $\widetilde{C}_{b}([a,+\infty) ; Y)$.

We also denote by $\widetilde{L}^{1}(0,+\infty ; Y)$ the space $L_{\text {loc }}^{1}(0,+\infty ; Y)$ endowed with the family of semi-norms $\left\{\|\cdot\|_{k, x^{*}} ; x^{*} \in L^{1}(0, k ; Y)^{*}, k=1,2, \ldots\right\}$, defined by $\|g\|_{k, x^{*}}=\left|x^{*}(g)\right|$. This space is also locally convex and separated. Moreover, the convergence in $\widetilde{L}^{1}(0,+\infty ; Y)$ is nothing but the weak convergence in $L^{1}(0, k ; Y)$ for $k=1,2, \ldots$.

In the sequel we need the next hypotheses:

$\left(H_{A}\right)$ the operator $A: D(A) \subseteq X \rightsquigarrow X$ is $m$-dissipative, $0 \in D(A), 0 \in A 0$ and there exists $\omega>0$ such that $A+\omega I$ is dissipative;

$\left(H_{B}\right)$ the operator $B: D(B) \subseteq Y \rightsquigarrow Y$ satisfies:

$\left(B_{1}\right) B$ is $m$-dissipative, $0 \in D(B), 0 \in B 0$ and there exists $\gamma>0$ such that $B+\gamma I$ is dissipative; 
$\left(B_{2}\right) B$ generates a compact semigroup;

$\left(B_{3}\right) B$ is of complete continuous type;

$\left(H_{F}\right)$ the function $F: \mathbb{R}_{+} \times C([-\tau, 0] ; \overline{D(A)}) \times C([-\tau, 0] ; \overline{D(B)}) \rightarrow X$ is continuous and satisfies:

$\left(F_{1}\right)$ there exists $\ell>0$ such that

$$
\|F(t, u, v)-F(t, \widetilde{u}, \widetilde{v})\| \leq \ell\left[\|u-\widetilde{u}\|_{C([-\tau, 0] ; X)}+\|v-\widetilde{v}\|_{C([-\tau, 0] ; Y)}\right]
$$

for each $t \in \mathbb{R}_{+}$, each $u, \widetilde{u} \in C([-\tau, 0] ; \overline{D(A)})$ and each $v, \widetilde{v} \in$ $C([-\tau, 0] ; \overline{D(B)})$;

$\left(F_{2}\right)$ there exists $m>0$ such that $\|F(t, 0,0)\| \leq m$ for each $t \in$ $[0,+\infty)$

$\left(H_{G}\right)$ the multi-function $G: \mathbb{R}_{+} \times C([-\tau, 0] ; \overline{D(A)}) \times C([-\tau, 0] ; \overline{D(B)}) \rightsquigarrow Y$ has nonempty, convex and weakly compact values and it is stronglyweakly u.s.c.;

$\left(G_{1}\right)$ with $\ell>0$ and $m>0$ given by $\left(F_{1}\right)$ and $\left(F_{2}\right)$, we have

$$
\|y\| \leq \ell\left[\|u\|_{C([-\tau, 0] ; X)}+\|v\|_{C([-\tau, 0] ; Y)]}\right]+m
$$

for each $u \in C([-\tau, 0] ; \overline{D(A)})$, each $v \in C([-\tau, 0] ; \overline{D(B)})$, each $y \in G(t, u, v)$ and each $t \geq 0$;

$\left(H_{c}\right)$ the constants $\ell>0, \omega>0$ and $\gamma>0$ satisfy the nonresonance condition $\ell<\frac{\omega \gamma}{\omega+\gamma}$;

$\left(H_{p}\right) p: C_{b}([-\tau,+\infty) ; \overline{D(A)}) \times C_{b}([-\tau,+\infty) ; \overline{D(B)}) \rightarrow C([-\tau, 0] ; \overline{D(A)})$ is continuous from the space $\widetilde{C}_{b}([-\tau,+\infty) ; \overline{D(A)}) \times \widetilde{C}_{b}([-\tau,+\infty) ; \overline{D(B)})$ to $C([-\tau, 0] ; \overline{D(A)})$ and satisfies:

$\left(p_{1}\right)$ for each $u \in C_{b}([-\tau,+\infty) ; \overline{D(A)})$ and each $v \in C_{b}([-\tau,+\infty)$; $\overline{D(B)})$, we have $\|p(u, v)\|_{C([-\tau, 0] ; X)} \leq\|u\|_{C_{b}([0,+\infty) ; X)} ;$

$\left(p_{2}\right)$ there exists $a>0$ such that for each $u, \widetilde{u} \in C_{b}([-\tau,+\infty) ; \overline{D(A)})$ and each $v, \widetilde{v} \in C_{b}([-\tau,+\infty) ; \overline{D(B)})$, we have

$$
\begin{aligned}
& \|p(u, v)-p(\widetilde{u}, \widetilde{v})\|_{C([-\tau, 0] ; X)} \\
& \quad \leq \max \left\{\|u-\widetilde{u}\|_{C_{b}([a,+\infty) ; X)},\|v-\widetilde{v}\|_{C_{b}([0,+\infty) ; Y)}\right\}
\end{aligned}
$$


$\left(H_{q}\right) q: C_{b}([-\tau,+\infty) ; X) \times C_{b}([-\tau,+\infty) ; \overline{D(B)}) \rightarrow C([-\tau, 0] ; \overline{D(B)})$ is continuous from $\widetilde{C}_{b}([-\tau,+\infty) ; \overline{D(A)}) \times \widetilde{C}_{b}([-\tau,+\infty) ; \overline{D(B)})$ to $C([-\tau, 0] ; \overline{D(B)})$ and satisfies:

$\left(q_{1}\right)$ for each $u \in C_{b}([-\tau,+\infty) ; X)$ and each $v \in C_{b}([-\tau,+\infty) ; \overline{D(B)})$, we have $\|q(u, v)\|_{C([-\tau, 0] ; Y)} \leq\|v\|_{C_{b}([0,+\infty) ; Y)}$;

$\left(q_{2}\right)$ for each $u, \widetilde{u} \in C_{b}([-\tau,+\infty) ; X)$ and $v, \widetilde{v} \in C_{b}([-\tau,+\infty) ; \overline{D(B)})$, we have $\|q(u, v)-q(\widetilde{u}, \widetilde{v})\|_{C([-\tau, 0] ; Y)} \leq \max \left\{\|u-\widetilde{u}\|_{C_{b}([0,+\infty) ; X)}\right.$, $\left.\|v-\widetilde{v}\|_{C_{b}([0,+\infty) ; Y)}\right\}$

$\left(q_{3}\right)$ for each bounded set $\mathcal{U}$ in $C_{b}([-\tau,+\infty) ; X)$ and each bounded $\mathcal{V}$ in $C_{b}([-\tau,+\infty) ; \overline{D(B)})$ which is relatively compact in $\widetilde{C}_{b}([\delta,+\infty) ; Y)$ for each $\delta \in(0,+\infty)$, the set $q(\mathcal{U}, \mathcal{V})$ is relatively compact in the space $C([-\tau, 0] ; Y)$.

Our main result is:

Theorem 3.1. If $\left(H_{A}\right),\left(H_{B}\right),\left(H_{F}\right),\left(H_{G}\right),\left(H_{p}\right),\left(H_{q}\right)$ and $\left(H_{c}\right)$ are satisfied, then (1.1) has at least one $C^{0}$-solution, $(u, v) \in C_{b}([-\tau,+\infty)$; $\overline{D(A)}) \times C_{b}([-\tau,+\infty) ; \overline{D(B)})$, satisfying

$$
\left\{\begin{array}{l}
\|u\|_{C_{b}([-\tau,+\infty) ; X)} \leq \frac{m}{\omega_{\bar{m}} \ell} \\
\|v\|_{C_{b}([-\tau,+\infty) ; Y)} \leq \frac{1}{\gamma-\ell} .
\end{array}\right.
$$

\section{Proof of Theorem 3.1}

Let $\varepsilon>0$, let $(f, g) \in C_{b}([0,+\infty) ; X) \times L^{\infty}(0,+\infty ; Y)$ and let us consider the system

$$
\left\{\begin{array}{lll}
u^{\prime}(t) \in A u(t)+f(t), & & t \in \mathbb{R}_{+}, \\
v^{\prime}(t) \in B v(t)+g(t), & & t \in \mathbb{R}_{+}, \\
u(t)=(1-\varepsilon) p(u, v)(t), & & t \in[-\tau, 0], \\
v(t)=(1-\varepsilon) q(u, v)(t), & & t \in[-\tau, 0] .
\end{array}\right.
$$

Lemma 4.1. If the hypotheses $\left(H_{A}\right),\left(B_{1}\right),\left(p_{1}\right),\left(p_{2}\right),\left(q_{1}\right)$ and $\left(q_{2}\right)$ are satisfied, then the system (4.1) has a unique $C^{0}$-solution $(u, v) \in C_{b}([-\tau, \infty)$; $\overline{D(A)}) \times C_{b}([-\tau, \infty) ; \overline{D(B)})$. 
If, in addition, $\|f\|_{\infty} \leq m$ and $\|g\|_{\infty} \leq m$, then we have

$$
\left\{\begin{array}{l}
\|u\|_{C_{b}([-\tau,+\infty) ; X)} \leq \frac{m}{\omega} \\
\|v\|_{C_{b}([-\tau,+\infty) ; Y)} \leq \frac{m}{\gamma} .
\end{array}\right.
$$

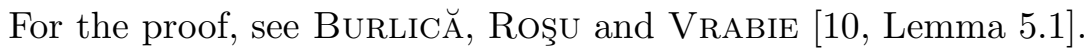

Idea of the proof of Theorem 3.1. Let us consider the approximate problem

$$
\begin{cases}u^{\prime}(t) \in A u(t)+F\left(t, u_{t}, v_{t}\right), & t \in \mathbb{R}_{+}, \\ v^{\prime}(t) \in B v(t)+g(t), & t \in \mathbb{R}_{+}, \\ g(t) \in \chi_{[0,1 / \varepsilon]}(t) G\left(t, u_{t}, v_{t}\right), & t \in \mathbb{R}_{+}, \\ u(t)=(1-\varepsilon) p(u, v)(t), & t \in[-\tau, 0], \\ v(t)=(1-\varepsilon) q(u, v)(t), & t \in[-\tau, 0],\end{cases}
$$

as well as the following two auxiliary systems

$$
\begin{cases}\widetilde{v}^{\prime}(t) \in B \widetilde{v}(t)+g(t), & t \in[0,+\infty), \\ \widetilde{v}(t)=(1-\varepsilon) q(u, \widetilde{v})(t), & t \in[-\tau, 0]\end{cases}
$$

and

$$
\begin{cases}\widetilde{u}^{\prime}(t) \in A \widetilde{u}(t)+F\left(t, \widetilde{u}_{t}, \widetilde{v}_{t}\right), & t \in[0,+\infty), \\ \widetilde{u}(t)=(1-\varepsilon) p(\widetilde{u}, \widetilde{v})(t), & t \in[-\tau, 0],\end{cases}
$$

where $\varepsilon \in(0,1)$ is arbitrary fixed. We will use a fixed point device described below.

Fix an arbitrary $(u, g) \in C_{b}([-\tau,+\infty) ; X) \times L^{\infty}(0,+\infty ; Y)$. Using VRABIE [24, Lemma 4.5], the problem (4.4) has a unique $C^{0}$-solution $\widetilde{v} \in$ $C_{b}([-\tau,+\infty) ; \overline{D(B)})$. Next, by Burlic $\breve{A}$ and Roşu [8, Theorem 3.1], the problem (4.5) has a unique $C^{0}$-solution $\widetilde{u} \in C_{b}([-\tau,+\infty) ; \overline{D(A)})$.

Now we define the multifunction

$\Gamma_{\varepsilon}: C_{b}([-\tau,+\infty) ; X) \times L^{\infty}(0,+\infty ; Y) \rightsquigarrow C_{b}([-\tau,+\infty) ; X) \times L^{\infty}(0,+\infty ; Y)$

by

$$
\begin{gathered}
\Gamma_{\varepsilon}(u, g):=\left\{(\widetilde{u}, \widetilde{g}) ; \widetilde{g} \in \widetilde{L}^{1}(0,+\infty ; Y), \widetilde{g}(t) \in G_{\varepsilon}\left(t, \widetilde{u}_{t}, \widetilde{v}_{t}\right)\right. \\
\text { a.e. for } t \in[0,+\infty)\},
\end{gathered}
$$


for each $(u, g) \in C_{b}([-\tau,+\infty) ; X) \times L^{\infty}(0,+\infty ; Y)$, where

$$
G_{\varepsilon}(t, u, v)=\chi_{[0,1 / \varepsilon]}(t) G(t, u, v),
$$

for each $(t, u, v) \in[0,+\infty) \times C([-\tau, 0] ; \overline{D(A)}) \times C([-\tau, 0] ; \overline{D(B)})$, and $\chi_{[0,1 / \varepsilon]}$ is the characteristic function of $[0,1 / \varepsilon]$. Obviously $(4.3)$ has a $C^{0}$ solution if and only if the multi-function $\Gamma_{\varepsilon}$ has a fixed point on a suitably defined set.

Finally, we consider a family $\left\{\left(u_{\varepsilon}, v_{\varepsilon}\right) ; \varepsilon \in(0,1)\right\}$ of $C^{0}$-solutions for the problem (4.3) and we show that we can pick up a sequence $\left(\left(u_{\varepsilon_{n}}, v_{\varepsilon_{n}}\right)\right)_{n}$, such that $\lim _{n} \varepsilon_{n}=0, \lim _{n}\left(u_{\varepsilon_{n}}, v_{\varepsilon_{n}}\right)=(u, v)$ in $C_{b}([-\tau,+\infty) ; X) \times \widetilde{C}_{b}([-\tau,+\infty) ; Y)$ and $(u, v)$ is a $C^{0}$-solution of (1.1).

We begin by showing that we can suitably define a nonempty, convex and compact set $\mathcal{K}_{\varepsilon}$ in the product space $C_{b}([-\tau,+\infty) ; X) \times L^{\infty}(0,+\infty ; Y)$ such that $\Gamma_{\varepsilon}$ maps $\mathcal{K}_{\varepsilon}$ into itself and has sequentially closed graph with respect to the strong topology on $C_{b}([-\tau,+\infty) ; X)$ and the locally convex topology on $\widetilde{L}^{1}(0,+\infty ; Y)$.

We will do this with the help of the next lemmas.

Lemma 4.2. Let $\left(H_{A}\right),\left(B_{1}\right),\left(B_{2}\right)$ in $\left(H_{B}\right),\left(H_{F}\right),\left(H_{p}\right),\left(H_{q}\right)$ and $\left(H_{c}\right)$ be satisfied, and let

$$
r=\frac{m \omega \gamma}{\omega \gamma-\ell(\omega+\gamma)}
$$

Then, for each $(u, g) \in C_{b}([-\tau,+\infty) ; X) \times L^{\infty}(0,+\infty ; Y)$ satisfying

$$
\left\{\begin{array}{l}
\|u\|_{C_{b}([-\tau,+\infty) ; X)} \leq \frac{r}{\omega} \\
\|g\|_{L^{\infty}(0,+\infty ; Y)} \leq r
\end{array}\right.
$$

the pair $(\widetilde{u}, \widetilde{v})$, where $\widetilde{v}$ is the unique $C^{0}$-solution of (4.4) and $\widetilde{u}$ the unique $C^{0}$-solution of (4.5), satisfies

$$
\left\{\begin{array}{l}
\|\widetilde{u}\|_{C_{b}([-\tau,+\infty) ; X)} \leq \frac{r}{\psi} \\
\|\widetilde{v}\|_{C_{b}([-\tau,+\infty) ; Y)} \leq \frac{r}{\gamma},
\end{array}\right.
$$

and

$$
\left\|F\left(t, \widetilde{u}_{t}, \widetilde{v}_{t}\right)\right\| \leq r
$$

for each $t \in[0,+\infty)$. 


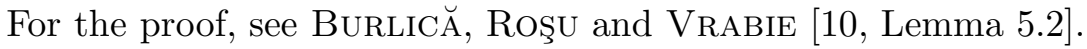

The lemma below is a continuity with respect to the data result and it

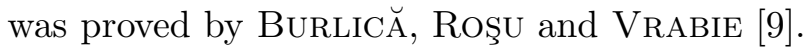

Lemma 4.3. Let $\left\{F_{n}: \mathbb{R}_{+} \times C([-\tau, 0] ; \overline{D(A)}) \rightarrow X ; n \in \mathbb{N}\right\}$ be a family of continuous functions satisfying:

$\left(h_{1}\right)$ there exists $\ell>0$ such that $\left\|F_{n}(t, x)-F_{n}(t, y)\right\| \leq \ell\|x-y\|_{C([-\tau, 0] ; X)}$ for each $n \in \mathbb{N}$, each $t \in[0,+\infty)$ and $x, y \in C([-\tau, 0] ; \overline{D(A)})$;

$\left(h_{2}\right)$ there exists $m>0$ such that $\left\|F_{n}(t, 0)\right\| \leq m$ for each $n \in \mathbb{N}$ and each $t \in[0,+\infty)$

$\left(h_{3}\right) \lim _{n} F_{n}(t, x)=F(t, x)$ uniformly for $t \in[0,+\infty)$ (for $t$ in bounded intervals in $[0,+\infty))$ and $x$ in bounded subsets in $C([-\tau, 0] ; \overline{D(A)})$.

Let $\left\{p_{n}: C_{b}([-\tau,+\infty) ; \overline{D(A)}) \rightarrow C([-\tau, 0] ; \overline{D(A)}) ; n \in \mathbb{N}\right\}$ be a family of functions satisfying:

$\left(h_{4}\right)$ for each $n \in \mathbb{N}$ and $u \in C_{b}([-\tau,+\infty) ; \overline{D(A)})$, we have $\left\|p_{n}(u)\right\|_{C([-\tau, 0] ; X)}$ $\leq\|u\|_{C_{b}([0,+\infty) ; X)}$

$\left(h_{5}\right)$ there exists $a>0$ such that for each $n \in \mathbb{N}$ and $u, \widetilde{u} \in C_{b}([-\tau,+\infty)$; $\overline{D(A)})$, we have $\left\|p_{n}(u)-p_{n}(\widetilde{u})\right\|_{C([-\tau, 0] ; X)} \leq\|u-\widetilde{u}\|_{C_{b}([a,+\infty) ; X)} ;$

$\left(h_{6}\right) \lim _{n} p_{n}(u)=p(u)$ uniformly for $u$ in bounded subsets in $C_{b}([-\tau,+\infty)$; $\overline{D(A)})$ (and $p$ is continuous from $\widetilde{C}_{b}([-\tau,+\infty) ; \overline{D(A)})$ to $C([-\tau, 0]$; $\overline{D(A)}))$.

Let us assume further that $A$ satisfies $\left(H_{A}\right)$ and $\ell<\omega$ holds true. Let $\left(u_{n}\right)_{n}$ be the sequence of $C^{0}$-solutions of the problem

$$
\begin{cases}u_{n}^{\prime}(t) \in A u_{n}(t)+F_{n}\left(t, u_{n_{t}}\right), & t \in[0,+\infty), \\ u_{n}(t)=p_{n}\left(u_{n}\right)(t), & t \in[-\tau, 0],\end{cases}
$$

whose existence and uniqueness is ensured by BuRLICĂ and Roşu [8, Theorem 3.1]. Then

$$
\lim _{n} u_{n}=u
$$

in $C_{b}([-\tau,+\infty) ; X)$ (in $\left.\widetilde{C}_{b}([-\tau,+\infty) ; X)\right)$, where $u$ is the $C^{0}$-solution of the limiting problem

$$
\begin{cases}u^{\prime}(t) \in A u(t)+F\left(t, u_{t}\right), & t \in[0,+\infty), \\ u(t)=p(u)(t), & t \in[-\tau, 0] .\end{cases}
$$


Lemma 4.4. Let us suppose that the hypotheses $\left(H_{A}\right),\left(H_{B}\right),\left(H_{F}\right)$, $\left(H_{G}\right),\left(H_{p}\right),\left(H_{q}\right)$ and $\left(H_{c}\right)$ are satisfied, and let $r>0$ be given by (4.7). Let $\rho=r / \omega$ and $K_{\varepsilon}:=K_{\rho} \times K_{r}$, where $K_{\rho}$ is the closed ball with center 0 and radius $\rho$ in $C_{b}([-\tau,+\infty) ; X)$ and $K_{r}$ is the closed ball with center 0 and radius $r$ in $L^{\infty}(0,+\infty ; Y)$ multiplied by $\chi_{[0,1 / \varepsilon]}$. Then the operator $\Gamma_{\varepsilon}$ defined by (4.6) maps $K_{\varepsilon}$ into itself and $\Gamma_{\varepsilon}$ has sequentially closed graph with respect to the norm topology on $C_{b}([-\tau,+\infty) ; X)$ and the locally convex topology on $\widetilde{L}^{1}(0,+\infty ; Y)$.

Proof. The operator

$\Gamma_{\varepsilon}: C_{b}([-\tau,+\infty) ; X) \times L^{\infty}(0,+\infty ; Y) \rightsquigarrow C_{b}([-\tau,+\infty) ; X) \times L^{\infty}(0,+\infty ; Y)$

is defined by

$\Gamma_{\varepsilon}(u, g):=\left\{(\widetilde{u}, \widetilde{g}) ; \widetilde{g} \in \widetilde{L}^{1}(0,+\infty ; Y), \widetilde{g}(t) \in G_{\varepsilon}\left(t, \widetilde{u}_{t}, \widetilde{v}_{t}\right)\right.$ a.e. for $\left.t \in[0,+\infty)\right\}$

where $G_{\varepsilon}\left(t, \widetilde{u}_{t}, \widetilde{v}_{t}\right)=\chi_{[0,1 / \varepsilon]}(t) G\left(t, \widetilde{u}_{t}, \widetilde{v}_{t}\right)$ and $\widetilde{u}$ is the unique $C^{0}$-solution of the problem (4.5) where $\widetilde{v}$ is the unique $C^{0}$-solution of the problem (4.4). If $(u, g) \in K_{\varepsilon}$, from Lemma 4.2 we deduce that the pair $(\widetilde{u}, \widetilde{v}) \in$ $C_{b}([-\tau, \infty) ; \overline{D(A)}) \times C_{b}([-\tau, \infty) ; \overline{D(B)})$ satisfies

$$
\|\widetilde{u}\|_{C_{b}([-\tau,+\infty) ; Y)} \leq \frac{r}{\omega}, \quad\|\widetilde{v}\|_{C_{b}([-\tau,+\infty) ; Y)} \leq \frac{r}{\gamma} .
$$

An appeal to $\left(G_{1}\right)$ shows that $\Gamma_{\varepsilon}$ maps $K_{\varepsilon}$ into itself.

To prove that $\Gamma_{\varepsilon}$ has sequentially closed graph with respect to the norm topology on $C_{b}([-\tau,+\infty) ; X)$ and the locally convex topology on $\widetilde{L}^{1}(0,+\infty ; Y)$, let $\left(\left(u_{n}, g_{n}\right)\right)_{n}$ be an arbitrary sequence in $K_{\varepsilon}$ and $\left(\widetilde{u}_{n}, \widetilde{g}_{n}\right) \in$ $\Gamma_{\varepsilon}\left(u_{n}, g_{n}\right)$ for each $n \in \mathbb{N}$. That means there exists $\widetilde{v}_{n} \in C_{b}([-\tau, \infty) ; \overline{D(B)})$, the unique $C^{0}$-solution for the problem

$$
\begin{cases}\widetilde{v}_{n}^{\prime}(t) \in B \widetilde{v}_{n}(t)+g_{n}(t), & t \in[0,+\infty), \\ \widetilde{v}_{n}(t)=(1-\varepsilon) q\left(u_{n}, \widetilde{v}_{n}\right)(t), & t \in[-\tau, 0],\end{cases}
$$

and $\widetilde{u}_{n} \in C_{b}([-\tau, \infty) ; \overline{D(A)})$, the unique $C^{0}$-solution for the problem

$$
\begin{cases}\widetilde{u}_{n}^{\prime}(t) \in A \widetilde{u}_{n}(t)+F\left(t, \widetilde{u}_{n_{t}}, \widetilde{v}_{n_{t}}\right), & t \in[0,+\infty), \\ \widetilde{u}_{n}(t)=(1-\varepsilon) p\left(\widetilde{u}_{n}, \widetilde{v}_{n}\right)(t), & t \in[-\tau, 0],\end{cases}
$$


for $n \in \mathbb{N}$. By the definition of $\Gamma_{\varepsilon}, \widetilde{g}_{n} \in \widetilde{L}^{1}(0,+\infty ; Y)$ and $\widetilde{g}_{n}(t) \in$ $G_{\varepsilon}\left(t, \widetilde{u}_{n_{t}}, \widetilde{v}_{n_{t}}\right)$ a.e. for $t \in[0,+\infty)$ and for each $n \in \mathbb{N}$. We suppose that

$$
\lim _{n}\left(u_{n}, g_{n}\right)=(u, g) \text { in } C_{b}([-\tau,+\infty) ; X) \times \widetilde{L}^{1}(0,+\infty ; Y)
$$

and

$$
\lim _{n}\left(\widetilde{u}_{n}, \widetilde{g}_{n}\right)=(\widetilde{u}, \widetilde{g}) \text { in } C_{b}([-\tau,+\infty) ; X) \times \widetilde{L}^{1}(0,+\infty ; Y)
$$

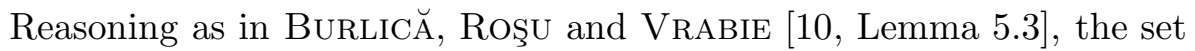
$\left\{\widetilde{v}_{n} ; n \in \mathbb{N}\right\}$ is relatively compact in $C_{b}([-\tau,+\infty) ; Y)$. Indeed, from (4.13), we get $\left\|\widetilde{v}_{n}(0)\right\| \leq\left\|\widetilde{v}_{n}\right\|_{C_{b}([-\tau,+\infty) ; Y)} \leq \frac{r}{\gamma}$ for each $n \in \mathbb{N}$ and, by Remark 2.1, the set $\left\{g_{n} ; n \in \mathbb{N}\right\}$ is uniformly integrable, so we are in the hypotheses of Theorem 2.2, wherefrom we obtain that $\left\{\widetilde{v}_{n} ; n \in \mathbb{N}\right\}$ is relatively compact in $C([\delta, k] ; Y)$ for $k=1,2, \ldots$ and $\delta \in(0, k)$. Since $\left\{u_{n} ; n \in \mathbb{N}\right\}$ is bounded in $C_{b}([-\tau,+\infty) ; X)$ and, from $\left(q_{3}\right),\left\{\widetilde{v}_{n}(0) ; n \in \mathbb{N}\right\}$ is relatively compact in $Y$, we can apply the second part of Theorem 2.2 , wherefrom $\left\{\widetilde{v}_{n} ; n \in \mathbb{N}\right\}$ is relatively compact in $C_{b}([0, k] ; Y)$ for $k=1,2, \ldots$ An appeal to condition $\left(q_{1}\right)$ shows that $\left\{\widetilde{v}_{n} ; n \in \mathbb{N}\right\}$ is relatively compact in $\widetilde{C}_{b}([-\tau,+\infty) ; Y)$.

That means that there exists $\widetilde{v} \in C_{b}([-\tau,+\infty) ; Y)$ such that on a subsequence of $\left(\widetilde{v}_{n}\right)_{n}$-denoted for simplicity by $\left(\widetilde{v}_{n}\right)_{n}$, we have

$$
\lim _{n} \widetilde{v}_{n}=\widetilde{v}
$$

in $\widetilde{C}_{b}([-\tau,+\infty) ; Y)$. In fact, we can prove a stronger condition, i.e. the convergence in $C_{b}([-\tau,+\infty) ; Y)$.

Since $g_{n} \in K_{r}$ which is weakly closed in $\widetilde{L}^{1}(0,+\infty ; Y)$ and $\lim _{n} g_{n}=g$ weakly in $\widetilde{L}^{1}(0,+\infty ; Y)$, we deduce that $g \in K_{r}$. From this relation combined with (4.18) and with the fact that $B$ is of complete continuous type, we obtain that $\widetilde{v}$ is $C^{0}$-solution of the problem $\widetilde{v}^{\prime}(t) \in B \widetilde{v}(t)+g(t), t \in[0,+\infty)$. Next, we fix $k_{\varepsilon} \in \mathbb{N}$, with $k_{\varepsilon} \geq 1+1 / \varepsilon$ and an arbitrary constant $\alpha>0$. The sequence $\left(\widetilde{v}_{n}\right)_{n}$ is convergent to $\widetilde{v}$ on $\left[0, k_{\varepsilon}\right]$, so there exists $n_{\varepsilon}(\alpha) \in \mathbb{N}$ such that $\left\|\widetilde{v}_{n}(t)-\widetilde{v}(t)\right\| \leq \alpha$ for $n \in \mathbb{N}, n \geq n_{\varepsilon}(\alpha)$ and $t \in\left[0, k_{\varepsilon}\right]$.

For a.e. $s \in\left[k_{\varepsilon}, t\right],\left\|g_{n}(s)-g(s)\right\|=0$ and, using (2.2), we deduce

$$
\begin{aligned}
\left\|\widetilde{v}_{n}(t)-\widetilde{v}(t)\right\| & \leq e^{-\gamma k_{\varepsilon}}\left\|\widetilde{v}_{n}\left(k_{\varepsilon}\right)-\widetilde{v}\left(k_{\varepsilon}\right)\right\|+\int_{k_{\varepsilon}}^{t} e^{-\gamma(t-s)}\left\|g_{n}(s)-g(s)\right\| d s \\
& \leq\left\|\widetilde{v}_{n}\left(k_{\varepsilon}\right)-\widetilde{v}\left(k_{\varepsilon}\right)\right\| \leq \alpha
\end{aligned}
$$


for $n \geq n_{\varepsilon}(\alpha)$ and $t \geq k_{\varepsilon}$. From this inequality and taken into account (4.16), we obtain that

$$
\lim _{n} \widetilde{v}_{n}=\widetilde{v}
$$

in $C_{b}([0,+\infty) ; Y)$ and so, in $C_{b}([-\tau,+\infty) ; Y)$. From $\left(q_{2}\right)$ we deduce that $\widetilde{v}(t)=(1-\varepsilon) q(u, \widetilde{v})(t)$ for each $t \in[-\tau, 0]$.

Since for each $n \in \mathbb{N}, \widetilde{u}_{n}$ is the $C^{0}$-solution of the problem (4.15) and, by $\left(F_{1}\right),\left(p_{1}\right)$ and $\left(p_{2}\right)$, the functions $F_{n}(t, \cdot):=F\left(t, \cdot, \widetilde{v}_{n_{t}}\right)$ and $p_{n}(\cdot):=$ $(1-\varepsilon) p\left(\cdot, \widetilde{v}_{n}\right)$ for $n \in \mathbb{N}$ and $t \in[0,+\infty)$, satisfy Lemma 4.3, we deduce that the limit function $\widetilde{u}=\lim _{n} \widetilde{u}_{n}$ is the unique $C^{0}$-solution of the problem

$$
\begin{cases}\widetilde{u}^{\prime}(t) \in A \widetilde{u}(t)+F\left(t, \widetilde{u}_{t}, \widetilde{v}_{t}\right), & t \in[0,+\infty), \\ \widetilde{u}(t)=(1-\varepsilon) p(\widetilde{u}, \widetilde{v})(t), & t \in[-\tau, 0] .\end{cases}
$$

Finally, let us prove that $\widetilde{g}(t) \in G_{\varepsilon}\left(t, \widetilde{u}_{t}, \widetilde{v}_{t}\right)$ a.e. $t \in[0, \infty)$. We have $\widetilde{g}_{n}(t) \in G_{\varepsilon}\left(t, \widetilde{u}_{n_{t}}, \widetilde{v}_{n_{t}}\right)$ a.e. $t \in[0, k]$ and $G_{\varepsilon}$ is strongly-weakly u.s.c.,

$$
\lim _{n} \widetilde{g}_{n}=\widetilde{g} \text { weakly in } L^{1}([0, k] ; Y) \text {, }
$$

$\lim _{n}\left(\widetilde{u}_{n_{t}}, \widetilde{v}_{n_{t}}\right)=\left(\widetilde{u}_{t}, \widetilde{v}_{t}\right)$ in $C([-\tau, 0] ; \overline{D(A)}) \times C([-\tau, 0] ; \overline{D(B)})$ a.e. $t \in[0, k]$,

for each $k=1,2, \ldots$. So, we are in the hypotheses of VRABIE [21, Theorem 3.1.2, p. 88], wherefrom we deduce that $\widetilde{g}(t) \in G_{\varepsilon}\left(t, \widetilde{u}_{t}, \widetilde{v}_{t}\right)$ a.e. $t \in$ $[0, k]$, for $k=1,2, \ldots$ and thus a.e. $t \in[0, \infty)$.

Lemma 4.5. Let us suppose that the hypotheses $\left(H_{A}\right),\left(H_{B}\right),\left(H_{F}\right)$, $\left(H_{G}\right),\left(H_{p}\right),\left(H_{q}\right)$ and $\left(H_{c}\right)$ are satisfied. Then, for each $\varepsilon \in(0,1)$, the set $\overline{\operatorname{conv} \Gamma_{\varepsilon}\left(K_{\varepsilon}\right)}$ is compact in the product space $C_{b}([-\tau,+\infty) ; X) \times \widetilde{L}^{1}(0,+\infty ; Y)$.

Proof. We begin by proving that the set $\Gamma_{\varepsilon}\left(K_{\varepsilon}\right)$ is relatively compact in the product space $C_{b}([-\tau,+\infty) ; X) \times \widetilde{L}^{1}(0,+\infty ; Y)$. Let $\left(\left(\widetilde{u}_{n}, \widetilde{g}_{n}\right)\right)_{n}$ be an arbitrary sequence in $\Gamma_{\varepsilon}\left(K_{\varepsilon}\right)$ and $\left(\left(u_{n}, g_{n}\right)\right)_{n} \in K_{\varepsilon}$ such that $\left(\widetilde{u}_{n}, \widetilde{g}_{n}\right) \in$ $\Gamma_{\varepsilon}\left(u_{n}, g_{n}\right)$ for $n \in \mathbb{N}$. So, $\widetilde{v}_{n}$ is the unique $C^{0}$-solution of the problem

$$
\begin{cases}\widetilde{v}_{n}^{\prime}(t) \in B \widetilde{v}_{n}(t)+g_{n}(t), & t \in[0,+\infty), \\ \widetilde{v}_{n}(t)=(1-\varepsilon) q\left(u_{n}, \widetilde{v}_{n}\right)(t), & t \in[-\tau, 0]\end{cases}
$$

and $\widetilde{u}_{n}$ is the unique $C^{0}$-solution of the problem

$$
\begin{cases}\widetilde{u}_{n}^{\prime}(t) \in A \widetilde{u}_{n}(t)+F\left(t, \widetilde{u}_{n_{t}}, \widetilde{v}_{n_{t}}\right), & t \in[0,+\infty), \\ \widetilde{u}_{n}(t)=(1-\varepsilon) p\left(\widetilde{u}_{n}, \widetilde{v}_{n}\right)(t), & t \in[-\tau, 0],\end{cases}
$$


for each $n \in \mathbb{N}$. Since $\left\{g_{n} ; n \in \mathbb{N}\right\}$ is bounded in $L^{\infty}(0,+\infty ; Y)$ we deduce that there exits $g \in L^{\infty}(0,+\infty ; Y)$ such that on a subsequence at least, we have $\lim _{n} g_{n}=g$ weakly in $\widetilde{L}^{1}(0, \infty ; Y)$. Reasoning as in Lemma 4.4, we deduce that there exists $\widetilde{v} \in C_{b}([-\tau,+\infty) ; Y)$ and a sub-subsequence of $\left(\widetilde{v}_{n}\right)_{n}$-denoted for simplicity again by $\left(\widetilde{v}_{n}\right)_{n}-$, such that $\lim _{n} \widetilde{v}_{n}=\widetilde{v}$ in $C_{b}([-\tau,+\infty) ; Y)$. Let $\widetilde{u} \in C_{b}([-\tau,+\infty) ; \overline{D(A)})$ the unique $C^{0}$-solution of the problem

$$
\begin{cases}\widetilde{u}^{\prime}(t) \in A \widetilde{u}(t)+F\left(t, \widetilde{u}_{t}, \widetilde{v}_{t}\right), & t \in[0,+\infty), \\ \widetilde{u}(t)=(1-\varepsilon) p(\widetilde{u}, \widetilde{v})(t), & t \in[-\tau, 0],\end{cases}
$$

with $\widetilde{v}$ as above. Reasoning as in Lemma 4.4, we deduce that on a subsequence at least, we have $\lim _{n} \widetilde{u}_{n}=\widetilde{u}$ in $C_{b}([-\tau,+\infty) ; X)$.

Since $\widetilde{g}_{n}(t) \in G_{\varepsilon}\left(t, \widetilde{u}_{n_{t}}, \widetilde{v}_{n_{t}}\right)$ a. e. for $t \in[0,+\infty)$ and for each $n \in \mathbb{N}$, by $\left(G_{1}\right)$, we deduce that, at least on a subsequence-denoted for simplicity by $\left(\widetilde{g}_{n}\right)_{n^{-}}$, we have

$$
\lim _{n} \widetilde{g}_{n}=\widetilde{g} \text { weakly in } \widetilde{L}^{1}(0, \infty ; Y)
$$

Furthermore, because the set $K_{r}$ is weakly closed in $\widetilde{L}^{1}(0,+\infty ; Y)$ we deduce that $\widetilde{g} \in K_{r}$. Let us observe that we have

$$
\widetilde{g}(t) \in G_{\varepsilon}\left(t, \widetilde{u}_{t}, \widetilde{v}_{t}\right)
$$

a.e. $t \in[0, \infty)$. Indeed, let us remind that $\widetilde{g}_{n}(t) \in G_{\varepsilon}\left(t, \widetilde{u}_{n_{t}}, \widetilde{v}_{n_{t}}\right)$ a.e. $t \in[0, k], G_{\varepsilon}$ is strongly-weakly u.s.c.,

$$
\begin{gathered}
\lim _{n} \widetilde{g}_{n}=\widetilde{g} \text { weakly in } L^{1}([0, k] ; Y), \\
\lim _{n}\left(\widetilde{u}_{n_{t}}, \widetilde{v}_{n_{t}}\right)=\left(\widetilde{u}_{t}, \widetilde{v}_{t}\right) \operatorname{in} C([-\tau, 0] ; X) \times C([-\tau, 0] ; Y) \text { a.e. } t \in[0, k],
\end{gathered}
$$

for each $k=1,2, \ldots$. So, by VRABIE [21, Theorem 3.1.2, p. 88], we obtain that $\widetilde{g}(t) \in G_{\varepsilon}\left(t, \widetilde{u}_{t}, \widetilde{v}_{t}\right)$ a.e. $t \in[0, k]$, for $k=1,2, \ldots$ and thus a.e. $t \in$ $[0, \infty)$. We conclude that $\Gamma_{\varepsilon}\left(K_{\varepsilon}\right)$ is relatively compact in $C_{b}([-\tau,+\infty) ; X) \times$ $L^{1}(0,+\infty ; Y)$ and thus, by Dunford and Schwartz [13, Theorem 6 , p. 416], $\overline{\operatorname{conv} \Gamma_{\varepsilon}\left(K_{\varepsilon}\right)}$ is compact in $C_{b}([-\tau,+\infty) ; X) \times \widetilde{L}^{1}(0,+\infty ; Y)$.

Now, we prove Theorem 3.1.

Proof. Let $\varepsilon \in(0,1)$ be arbitrary but fixed and let $\mathcal{K}_{\varepsilon}=\overline{\operatorname{conv} \Gamma_{\varepsilon}\left(K_{\varepsilon}\right)}$. By Lemma 4.5, it follows that the operator $\Gamma_{\varepsilon}: \mathcal{K}_{\varepsilon} \rightsquigarrow \mathcal{K}_{\varepsilon}$ has convex and 
compact values. Moreover, by Lemma 4.4, the graph of $\Gamma_{\varepsilon}$ is sequentially closed. Since, in a Banach space, the weak closure of a weakly relatively compact set coincides with its weak sequential closure - see EDWARDS [14, Theorem 8.12.1, p. 549] -, using a standard diagonal process, we deduce that the graph of $\Gamma_{\varepsilon}$ is even closed in $C_{b}([-\tau,+\infty) ; X) \times \widetilde{L}^{1}(0,+\infty ; Y)$. From Theorem 2.3, we deduce that $\Gamma_{\varepsilon}$ has at least on fixed point $\left(u_{\varepsilon}, g_{\varepsilon}\right)$. Clearly this means that the approximate problem (4.3) has at least one solution $\left(u_{\varepsilon}, v_{\varepsilon}\right)$. For each $\varepsilon \in(0,1)$, fix such a solution and consider the set $\left\{\left(u_{\varepsilon}, v_{\varepsilon}\right) ; \varepsilon \in(0,1)\right\}$.

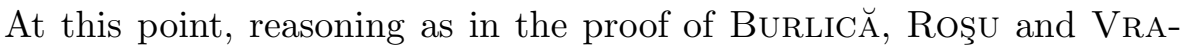
BIE [10, Theorem 3.1], we deduce that the set $\left\{\left(u_{\varepsilon}, v_{\varepsilon}\right) ; \varepsilon \in(0,1)\right\}$ is relatively compact in $\widetilde{C}_{b}([-\tau,+\infty) ; X) \times \widetilde{C}_{b}([-\tau,+\infty) ; Y)$. Indeed, let $\varepsilon_{n} \downarrow 0$ and let $\left(\left(u_{n}, v_{n}\right)\right)_{n}$ be a sequence of solutions for

$$
\left\{\begin{array}{lll}
u_{n}^{\prime}(t) \in A u_{n}(t)+F\left(t, u_{n_{t}}, v_{n_{t}}\right), & & t \in \mathbb{R}_{+}, \\
v_{n}^{\prime}(t) \in B v_{n}(t)+g_{n}(t), & & t \in \mathbb{R}_{+}, \\
g_{n}(t) \in \chi_{\left[0,1 / \varepsilon_{n}\right]}(t) G\left(t, u_{n_{t}}, v_{n_{t}}\right), & & t \in \mathbb{R}_{+}, \\
u_{n}(t)=\left(1-\varepsilon_{n}\right) p\left(u_{n}, v_{n}\right)(t), & & t \in[-\tau, 0], \\
v_{n}(t)=\left(1-\varepsilon_{n}\right) q\left(u_{n}, v_{n}\right)(t), & & t \in[-\tau, 0] .
\end{array}\right.
$$

Arguing as in Lemma 4.5, we deduce that the set $\left\{g_{n} ; n \in \mathbb{N}\right\}$ is weakly relatively compact in $L^{1}(0, k ; Y)$, for $k=1,2, \ldots$ and the set $\left\{v_{n} ; n \in\right.$ $\mathbb{N}\}$ is relatively compact in $\widetilde{C}_{b}([-\tau,+\infty) ; Y)$. So, at least on a subsequence, we have both $\lim _{n} g_{n}=g$ weakly in $\widetilde{L}^{1}(0, \infty ; Y), \lim _{n} v_{n}=v$ in $\widetilde{C}_{b}([-\tau,+\infty) ; Y)$ and the function $v$ satisfies $v^{\prime}(t) \in B v(t)+g(t)$ for each $t \in[0,+\infty)$. Using $\left(H_{F}\right),\left(p_{1}\right),\left(p_{2}\right)$ and the continuity property of $p$ we deduce that we can apply Theorem $4.3, \widetilde{C}_{b}$-continuity part, with $F_{n}(t, \cdot):=F\left(t, \cdot, v_{n_{t}}\right)$ and $p_{n}(\cdot):=(1-\varepsilon) p\left(\cdot, v_{n}\right)$ for $n \in \mathbb{N}$ and $t \in[0,+\infty)$. So, at least on a subsequence, we have $\lim _{n} u_{n}=u$ in $\widetilde{C}_{b}([-\tau,+\infty) ; X)$ where

$$
\begin{cases}u^{\prime}(t) \in A u(t)+F\left(t, u_{t}, v_{t}\right), & t \in \mathbb{R}_{+}, \\ u(t)=p(u, v)(t), & t \in[-\tau, 0] .\end{cases}
$$

Since $G$ is strongly-weakly u.s.c. and $g_{n}(t) \in \chi_{\left[0,1 / \varepsilon_{n}\right]}(t) G\left(t, u_{n_{t}}, u_{n_{t}}\right)$ a.e. for $t \in[0,+\infty)$, from VRABIE [21, Theorem 3.1.2, p. 88] we get $g(t) \in$ $G\left(t, u_{t}, v_{t}\right)$ a.e. $t \in[0, \infty)$. Finally, from the continuity property of $q$ we get $v(t)=q(u, v)(t)$ for each $t \in[-\tau, 0]$ and this completes the proof. 


\section{An example}

Let $\Omega$ be a nonempty, bounded domain in $\mathbb{R}^{d}, d \geq 2$, with $C^{1}$ boundary $\Gamma$, let $\tau>0, \omega>0$, let $Q=\mathbb{R}_{+} \times \Omega, \Sigma=\mathbb{R}_{+} \times \Gamma, \Omega_{\tau}=[-\tau, 0] \times \Omega$ and let $\Delta$ be the Laplace operator in the sense of distributions over $\Omega$. Let $\varphi: D(\varphi) \subseteq$ $\mathbb{R} \rightsquigarrow \mathbb{R}$ and $\psi: D(\psi) \subseteq \mathbb{R} \rightsquigarrow \mathbb{R}$ be maximal-monotone operators with $0 \in \varphi(0), 0 \in \psi(0)$, let $F: \mathbb{R}_{+} \times C\left([-\tau, 0] ; L^{1}(\Omega)\right) \times C\left([-\tau, 0] ; L^{1}(\Omega)\right) \rightarrow$ $L^{1}(\Omega)$ be continuous and $g_{i}: \mathbb{R}_{+} \times C\left([-\tau, 0] ; L^{1}(\Omega)\right) \times C\left([-\tau, 0] ; L^{1}(\Omega)\right) \rightarrow$ $L^{1}(\Omega), i=1,2$, two given functions such that $g_{1}$ is l.s.c, $g_{2}$ is u.s.c and $g_{1}(t, u, v)(x) \leq g_{2}(t, u, v)(x)$, for each $(t, u, v) \in \mathbb{R}_{+} \times C\left([-\tau, 0] ; L^{1}(\Omega)\right) \times$ $C\left([-\tau, 0] ; L^{1}(\Omega)\right)$ and a.e. $x \in \Omega$. Let $a>0$, let $\mu$ be a positive $\sigma$-finite and complete measure on the class of Borel measurable sets in $[a,+\infty)$, $k \in L^{1}(a,+\infty ; \mu, \mathbb{R})$ be a nonnegative function with $\|k\|_{L^{1}(a,+\infty ; \mu, \mathbb{R})} \leq 1$ and let $W: C\left([-\tau, 0] ; L^{1}(\Omega)\right) \rightarrow \mathbb{R}_{+}$be nonexpansive with $W(0)=0$. We consider the following system:

$$
\begin{cases}\frac{\partial u}{\partial t}(t, x)=\Delta \varphi(u(t, x))-\omega u(t, x)+F\left(t, u_{t}, v_{t}\right)(x), & (t, x) \in Q, \\ \frac{\partial v}{\partial t}(t, x)=\Delta \psi(v(t, x))-\gamma v(t, x)+g(t)(x), & (t, x) \in Q \\ g(t) \in G\left(t, u_{t}, v_{t}\right), & t \in \mathbb{R}_{+}, \\ \varphi(u(t, x))=0, \psi(v(t, x))=0, & (t, x) \in \Sigma, \\ u(t, x)=\int_{a}^{\infty} k(s) W(v(t+s, x)) u(t+s, x) d \mu(s), & (t, x) \in \Omega_{\tau} \\ v(t, x)=v(t+T, x), & (t, x) \in \Omega_{\tau}\end{cases}
$$

where

$$
G: \mathbb{R}_{+} \times C\left([-\tau, 0] ; L^{1}(\Omega)\right) \times C\left([-\tau, 0] ; L^{1}(\Omega)\right) \rightsquigarrow L^{1}(\Omega)
$$

is defined by

$G(t, u, v):=\left\{h \in L^{1}(\Omega) ; g_{1}(t, u, v)(x) \leq h(x) \leq g_{2}(t, u, v)(x)\right.$, a.e. $\left.x \in \Omega\right\}$.

If $\theta: D(\theta) \subseteq \mathbb{R} \rightsquigarrow \mathbb{R}$ is monotone with $0 \in \theta(0)$ and $u: \Omega \rightarrow D(\theta)$, we denote by

$$
\mathcal{S}_{\theta}(u)=\left\{v \in L^{1}(\Omega) ; v(x) \in \theta(u(x)), \text { a.e. for } x \in \Omega\right\} .
$$

The first part of the result below was proved by Brezis and STrauss [4] while the second was proved by BADII, DíAz and TESEI [1]. 
Theorem 5.1. Let $\Omega$ be a nonempty, bounded and open subset in $\mathbb{R}^{d}$ with $C^{1}$ boundary $\Gamma$ and let $\theta: D(\theta) \subseteq \mathbb{R} \rightsquigarrow \mathbb{R}$ be maximal monotone with $0 \in D(\theta)$ and $0 \in \theta(0)$.

(i) Then the operator $\Delta \theta: D(\Delta \theta) \subseteq L^{1}(\Omega) \rightsquigarrow L^{1}(\Omega)$, defined by

$$
\left\{\begin{array}{l}
D(\Delta \theta)=\left\{u \in L^{1}(\Omega) ; \exists v \in \mathcal{S}_{\theta}(u) \cap W_{0}^{1,1}(\Omega), \Delta v \in L^{1}(\Omega)\right\} \\
\Delta \theta(u)=\left\{\Delta v ; v \in \mathcal{S}_{\theta}(u) \cap W_{0}^{1,1}(\Omega)\right\} \cap L^{1}(\Omega) \text { for } u \in D(\Delta \theta),
\end{array}\right.
$$

is m-dissipative on $L^{1}(\Omega)$.

(ii) If, in addition, $\theta: \mathbb{R} \rightarrow \mathbb{R}$ is continuous on $\mathbb{R}$ and $C^{1}$ on $\mathbb{R} \backslash\{0\}$ and there exist two constants $C>0$ and $\alpha>0$ if $d \leq 2$ and $\alpha>(d-2) / d$ if $d \geq 3$ such that $\theta^{\prime}(r) \geq C|r|^{\alpha-1}$ for each $r \in \mathbb{R} \backslash\{0\}$, then $\Delta \theta$ generates a compact semigroup.

For a sufficient condition in order that the semigroup generated by $\Delta \theta$ maps weakly compact sets in $L^{1}(\Omega)$ into compact sets in $L^{1}(\Omega)$ for $t>0$, see DíAZ and VRABIE [11].

Before proceeding to the statement of the main result of this section, let us define the multifunction

$$
G: \mathbb{R}_{+} \times C\left([-\tau, 0] ; L^{1}(\Omega)\right) \times C\left([-\tau, 0] ; L^{1}(\Omega)\right) \rightsquigarrow L^{1}(\Omega)
$$

by $G(t, u, v)(x):=\left[g_{1}(t, u, v)(x), g_{2}(t, u, v)(x)\right]$, for each $t \in \mathbb{R}_{+}, x \in \Omega$ and $u, v \in C\left([-\tau, 0] ; L^{1}(\Omega)\right)$.

Theorem 5.2. Let $\Omega$ be a nonempty, bounded and open subset in $\mathbb{R}^{d}$, $d \geq 1$, with $C^{1}$ boundary $\Gamma$ and let $\varphi: D(\varphi) \subseteq \mathbb{R} \rightsquigarrow \mathbb{R}$ and $\psi: D(\psi) \subseteq$ $\mathbb{R} \rightsquigarrow \mathbb{R}$ be maximal monotone operators with $0 \in D(\varphi), 0 \in D(\psi), 0 \in \varphi(0)$ and $0 \in \psi(0)$. Let $F: \mathbb{R}_{+} \times C\left([-\tau, 0] ; L^{1}(\Omega)\right) \times C\left([-\tau, 0] ; L^{1}(\Omega)\right) \rightarrow$ $L^{1}(\Omega)$ be continuous, let $g_{i}: \mathbb{R}_{+} \times C\left([-\tau, 0] ; L^{1}(\Omega)\right) \times C\left([-\tau, 0] ; L^{1}(\Omega)\right) \rightarrow$ $L^{1}(\Omega), i=1,2$, be two given functions such that $g_{1}(t, u, v)(x) \leq g_{2}(t, u, v)(x)$, for each $(t, u, v) \in \mathbb{R}_{+} \times C\left([-\tau, 0] ; L^{1}(\Omega)\right) \times C\left([-\tau, 0] ; L^{1}(\Omega)\right)$ and a.e. $x \in \Omega$. Let $\mu$ be a positive $\sigma$-finite and complete measure defined on the class of Borel measurable sets in $[a,+\infty)$, let $k \in L^{1}(a,+\infty ; \mu, \mathbb{R})$ be nonnegative and let $W: C\left([-\tau, 0] ; L^{1}(\Omega)\right) \rightarrow \mathbb{R}_{+}$. Let us assume that:

$\left(h_{1}\right) \psi: \mathbb{R} \rightarrow \mathbb{R}$ is continuous on $\mathbb{R}$ and $C^{1}$ on $\mathbb{R} \backslash\{0\}$ and there exist two constants $C>0$ and $\alpha>0$ if $d \leq 2$ and $\alpha>(d-2) / d$ if $d \geq 3$ such that $\psi^{\prime}(r) \geq C|r|^{\alpha-1}$ for each $r \in \mathbb{R} \backslash\{0\}$; 
$\left(h_{2}\right)$ there exist $\ell>0$ and $m>0$ such that

$$
\begin{gathered}
\|F(t, u, v)-F(t, \widetilde{u}, \widetilde{v})\|_{L^{1}(\Omega)} \leq \ell\left[\|u-\widetilde{u}\|_{C\left([-\tau, 0] ; L^{1}(\Omega)\right)}\right. \\
\left.+\|v-\widetilde{v}\|_{C\left([-\tau, 0] ; L^{1}(\Omega)\right)}\right], \\
\|y\|_{L^{1}(\Omega)} \leq \ell\left[\|u\|_{C\left([-\tau, 0] ; L^{1}(\Omega)\right)}+\|v\|_{C\left([-\tau, 0] ; L^{1}(\Omega)\right)}\right]+m, \\
\|F(t, 0,0)\|_{L^{1}(\Omega)} \leq m,
\end{gathered}
$$

for each $(t, u, v),(t, \widetilde{u}, \widetilde{v}) \in \mathbb{R}_{+} \times C\left([-\tau, 0] ; L^{1}(\Omega)\right) \times C\left([-\tau, 0] ; L^{1}(\Omega)\right)$ and for each $y \in G(t, u, v)$.

$\left(h_{3}\right) g_{1}$ is l.s.c and $g_{2}$ is u.s.c;

$\left(h_{4}\right)$ there exist $\ell_{1}>0$ and $m_{1}>0$ such that for $i=1,2$ and for each $(t, u, v) \in \mathbb{R}_{+} \times C\left([-\tau, 0] ; L^{1}(\Omega)\right) \times C\left([-\tau, 0] ; L^{1}(\Omega)\right)$ we have

$$
\left\|g_{i}(t, u, v)\right\| \leq \ell_{1}\left[\|u\|_{C\left([-\tau, 0] ; L^{1}(\Omega)\right)}+\|v\|_{C\left([-\tau, 0] ; L^{1}(\Omega)\right)}\right]+m_{1} ;
$$

$\left(h_{5}\right)\|k\|_{L^{1}(a,+\infty ; \mu, \mathbb{R})} \leq 1$

$\left(h_{6}\right)|W(v)-W(\widetilde{v})| \leq\|v-\widetilde{v}\|_{C\left([-\tau, 0] ; L^{1}(\Omega)\right)}$, for each $v, \widetilde{v} \in C\left([-\tau, 0] ; L^{1}(\Omega)\right) ;$

$\left(h_{7}\right) W(0)=0$.

Let us assume also that $\left(H_{c}\right)$ is satisfied. Then, (5.1) has at least one $C^{0}$ solution.

Proof. The problem (5.1) can be rewritten as an abstract one of the form (1.1). Since $g_{1}$ is l.s.c., $g_{2}$ is u.s.c. and both have sublinear growth, we conclude that $G$ is strongly-weakly u.s.c. with nonempty, convex and weakly compact values. So, all hypotheses of the Theorem 3.1 are satisfied.

\section{REFERENCES}

1. BADII, M.; DÍAZ, J. I.; TESEI, A. - Existence and attractivity for a class of degenerate functional parabolic problems, Rend. Sem. Mat. Univ. Padova, 78 (1987), 109-124.

2. BARAs, P. - Compacité de l'opérateur $f \mapsto u$ solution d'une équation non linéaire $(d u / d t)+A u \ni f$, C. R. Acad. Sci. Paris Sér. A-B, 286 (1978), A1113-A1116. 
3. Barbu, V. - Nonlinear Differential Equations of Monotone Type in Banach Spaces, Springer Monographs in Mathematics, Springer, New York, 2010.

4. BrÉzIS, H.; Strauss, W.A. - Semi-linear second-order elliptic equations in $L^{1}$, J. Math. Soc. Japan, 25 (1973), 565-590.

5. Burlic $\breve{A}$, M. - Viability for multi-valued semilinear reaction-diffusion systems, Ann. Acad. Rom. Sci. Ser. Math. Appl., 2 (2010), 3-24.

6. Burlică, M.; Roşu, D. - A viability result for semilinear reaction-diffusion systems, An. Ştiinţ. Univ. "Al.I. Cuza" Iaşi. Mat. (N.S.), 54 (2008), 361-382.

7. Burlic $\breve{A}$, M.; Roşu, D. - The initial value and the periodic problems for a class of reaction-diffusion systems, Dyn. Contin. Discrete Impuls. Syst. Ser. A Math. Anal., 15 (2008), 427-444.

8. Burlic $\breve{,}$, M.; RoşU, D. - A class of nonlinear delay evolution equations with nonlocal initial conditions, Proc. Amer. Math. Soc., in print.

9. Burlic $\breve{a}$, M.; Roşu, D.; Vrabie, I. I. - Continuity with respect to the data for a delay evolution equation with nonlocal initial conditions, Lib. Math. (N.S.), 32 (2012), $39-50$.

10. Burlică, M.; Roşu, D.; VRabie, I. I. - Abstract reaction-diffusion systems with nonlocal initial conditions, submitted.

11. DíAZ, J. I.; VRABie, I. I. - Propriétés de compacité de l'opérateur de Green généralisé pour l'équation des milieux poreux, C. R. Acad Sci. Paris Sér. I Math., 309 (1989), $221-223$.

12. DíAz, J. I.; VRABIE, I. I. - Existence for reaction-diffusion systems. A compactness method approach, J. Math. Anal. Appl., 188 (1994), 521-540.

13. Dunford, N.; Schwartz, J.T. - Linear Operators. I, General Theory, Pure and Applied Mathematics, Vol. 7, Interscience Publishers, Inc., New York; Interscience Publishers, Ltd., London, 1958.

14. Edwards, R. E. - Functional Analysis. Theory and Applications, Holt, Rinehart and Winston, New York-Toronto-London, 1965.

15. Glicksberg, I. L. - A further generalization of the Kakutani fixed point theorem, with application to Nash equilibrium points, Proc. Amer. Math. Soc., 3 (1952), 170 174 .

16. Hale, J. - Theory of functional differential equations, Applied Mathematical Sciences, Vol. 3, Springer-Verlag, New York-Heidelberg, 1977.

17. Necula, M.; VRabie, I. I. - A viability result for a class of fully nonlinear reactiondiffusion systems, Nonlinear Anal., 69 (2008), 1732-1743.

18. Paicu, A.; VRabie, I. I. - A class of nonlinear evolution equations subjected to nonlocal initial conditions, Nonlinear Anal., 72 (2010), 4091-4100.

19. RoşU, D. - Viability for nonlinear multi-valued reaction-diffusion systems, NoDEA Nonlinear Differential Equations Appl., 17 (2010), 479-496. 
20. Roşu, D. - Viability for a nonlinear multi-valued system on locally closed graph, An. Ştiinţ. Univ. "Al. I. Cuza" Iaşi. Mat. (N.S.), 56 (2010), 343-362.

21. VRABie, I. I. - Compactness methods for nonlinear evolutions, Second edition, Pitman Monographs and Surveys in Pure and Applied Mathematics, 75, Longman Scientific \& Technical, Harlow; copublished in the United States with John Wiley \& Sons, Inc., New York, 1995.

22. VRABIE, I. I. - Existence for nonlinear evolution inclusions with nonlocal retarded initial conditions, Nonlinear Anal., 74 (2011), 7047-7060.

23. VRABIE, I. I. - Existence in the large for nonlinear delay evolution inclusions with nonlocal initial conditions, J. Funct. Anal., 262 (2012), 1363-1391.

24. VRABIE, I. I. - Nonlinear retarded ecolution equations with nonlocal initial conditions, Dynam. Systems Appl., 21 (2012), 417-439.

25. VRABIE, I. I. - Global solutions for nonlinear delay evolution inclusions with nonlocal initial conditions, Set-Valued Var. Anal., 20 (2012), 477-497.

Received: 15.XII.2012

Accepted: 1.III.2013

Department of Mathematics and Informatics, "G. Asachi" Technical University, Iaşi, 700506 , ROMANIA

monicaburlica@yahoo.com rosudaniela100@yahoo.com 